REVISTA

MEXICANA DE

ECONOMÍA Y

FINANZAS

REMEF

(THE MEXICAN JOURNAL OF

ECONOMICS AND FINANCE)
Revista Mexicana de Economía y Finanzas, Nueva Época

Volumen 16 Número 4, Octubre - Diciembre 2021, pp. 1-16, e509

DOI: https://doi.org/10.21919/remef.v16i4.509

(Recibido: 2/junio/2020, aceptado: 29/octubre/2020, publicado: 7/abril/2021)

\title{
Metodología de estimación agregada "gruesa" de los ingresos fiscales
}

\author{
Carlos Ortega-Laurel ${ }^{1}$ - Universidad Autónoma Metropolitana, México \\ Víctor Amaury Simental-Franco - Universidad del Valle de México, México
}

El objetivo de la presenta investigación es aportar a las subáreas de estimaciones fiscales-tributarias del área de finanzas públicas, una "metodología de estimación gruesa" aprovechando la estadística histórica de los ingresos, es el objetivo. A través del método de caso, se realiza el análisis y discusión de experiencias y situaciones fiscales de la Ciudad de México. Como resultado se obtiene la metodología, apoyada en el arreglo polinómico para funciones tabulares de recaudación por Interpolación con diferencias divididas de Newton. Se recomienda el uso de la metodología para la proyección y el análisis de los ingresos fiscales-tributarios en "entes" con autosuficiencia financiera. Se ubica como la principal limitación para la aplicación de la metodología, que la recaudación está centralizada y no hay autosuficiencia financiera en diversos "entes". Esta investigación es original dado que aporta una metodología de trabajo al área de estimaciones fiscales de las finanzas públicas. Se concluye que, es factible utilizar la metodología propuesta para realizar estimaciones de los ingresos fiscalestributarios, considerando como dato la estadística histórica de tales, que ha internalizado los efectos de las variables económicas.

Clasificación JEL: K34

Palabras clave: Metodología de estimación, funciones tabulares de recaudación, proyección de ingresos fiscales, análisis de los ingresos tributarios, estimaciones de las finanzas públicas.

\section{Methodology for the "Gross" Aggregate Estimation of Tax Revenues}

The objective of this research is to provide the fiscal-tax estimates subareas of the public finance area with a "rough estimation methodology" taking advantage of the historical statistics of income. Through the case method, the analysis and discussion of experiences and fiscal situations in Mexico City is carried out. As a result, the methodology is obtained, supported by the polynomial arrangement for tabular collection functions by Newton's interpolation with divided differences. The use of the methodology for the projection and analysis of fiscal-tax revenues in "entities" with financial self-sufficiency is recommended. The main limitation for the application of the methodology is that the tax collection is centralized and there is no financial self-sufficiency in various "entities". This research is original since it provides a work methodology to the fiscal estimates area of public finances. It is concluded that it is feasible to use the proposed methodology to make estimates of fiscaltax revenues, considering as data the historical statistics of such, which has internalized the effects of the economic variables.

JEL Classification: K34

Keywords: Estimation methodology, tabular collection functions, tax revenue projection, analysis of tax revenues, estimates of public finances.

\footnotetext{
${ }^{1}$ Autor de correspondencia. Email: c.ortega@correo.ler.uam.mx; ORCID: https://orcid.org/0000-0001-6072-8480

*Sin Fuente de financiamiento para el desarrollo de la investigación
} 


\section{Introducción}

Los ingresos tributarios es el área de las finanzas públicas que ha alcanzado un menor nivel de desarrollo en cuanto a técnicas y métodos de trabajo, esto es, metodologías que habiliten el obtener su principal producto: la proyección, el análisis de los ingresos tributarios y las estimaciones (Martín, 2009).

Se ubica esta problemática y se señala como razones del escaso desarrollo la índole diversa y compleja de las materias involucradas, además de la necesidad de trabajar con un futuro en cuya especulación, ideación, diagramación y verificación se tiene realmente muy poca o nula injerencia, y el predominio de la idea, en los ambientes profesional e institucionales que lo realmente importante es el gasto, asunto que comprensiblemente se torna mucho más interesante e importante para los actores toda vez que define el destino de los recursos, a donde irá a parar el dinero que se recauda, sin importar, inapropiadamente, el cálculo de los recursos tributarios, fuente que sustenta el que sea factible obtener los recursos que permitirán hacer frente a dicho gasto, y se le soslaya como un dato más (Martín, 2009).

Es en este sentido, dado que se identifica que la estimación de los ingresos tributarios tiene una explicación a la altura de la importancia que se le otorga al gasto, por ser el sostén esencial y la contracara justamente de todos los gastos públicos, se considera importante contribuir al desarrollo de metodologías de trabajo para el área disciplinar que habiliten el poder explicar de manera agregada el valor estimado de los recursos tributarios para los proyectos de Ley de ingresos.

Por lo anterior, en este trabajo, se traza como objetivo aportar a las subáreas de estimaciones tributarias del área de finanzas públicas, una técnica y método de trabajo: "metodología", que permita la estimación agregada ("gruesa"), usando como dato-insumo la estadística histórica de los ingresos tributarios.

Para esta investigación, se desarrolla como hipótesis exponer que es factible estimar y describir la recaudación a través de la estadística propia de la recaudación histórica, que ha internalizado los efectos de las variables económicas y otros.

\section{Estado del arte}

La estimación de los ingresos tributarios es el área de las finanzas públicas que ha alcanzado un menor nivel de desarrollo, substancialmente en cuanto al conjunto de técnicas y métodos de trabajo, "las metodologías" que permiten obtener el principal producto del área, es decir, la proyección y el análisis de los ingresos tributarios, que están en consonancia con la estructura reglamentaria nacional (Martín, 2009), (Jarach, 2013).

Representando lo anterior un problema de investigación, con el presente trabajo se pretende contribuir con un aporte a científico, esto es, una metodología que permita obtener el principal producto del área, es decir, la proyección y el análisis de los ingresos fiscales - tributarios al área disciplinar.

En la frontera del conocimiento, de manera incipiente asociados secundariamente con la línea disciplinar, existen trabajos precedentes que plantean que para estimar recaudación lo propio es 
realizar las estimaciones concepto por concepto que se busca estimar, haciendo la consideración de tal como un silo conceptual a estimar (OCDE et al., 2020). En este sentido son ubicables propuestas de estimación de la recaudación de conceptos puntuales, como el método directo de proyección de los ingresos tributarios (Martín, 2005), la estimación del potencial del impuesto a la renta en América Latina (Rossignolo, 2012); la estimación de la recaudación del impuesto sobre transacciones financieras: el caso español (Contreras y Contreras, 2015); la estimación del incumplimiento en el IVA, Años 2000 a 2004 (Salim et al., 2005); la estimación de la recaudación fiscal derivada del consumo de tabaco en España: estimaciones a partir de un modelo de adicción racional (Gracia y Chueca, 2000); la estimación de la recaudación del impuesto al hospedaje en el estado de Quintana Roo, México (Puls, 2014) o incluso comparativos entre dos conceptos, como lo es el trabajo de (Andino y Parra, 2007) sobre la estimación de la brecha de recaudación del IVA e impuesto a la renta de las sociedades por industria. Si bien las investigaciones en la literatura son particularmente útiles cuando lo que se busca es la realización de la estimación de algún concepto en lo particular, en el que se considere de manera exhaustiva las variables endógenas y exógenas al modelo para el concepto en análisis, esto no es particularmente útil cuando los conceptos a estimar, son todos y cada uno de los conceptos recaudables, que pueden ser algunos cientos dependiendo de la entidad, y por tanto al hacerse una labor titánica en el caso de que se decida estimar concepto por concepto, lo prudente es se busque obtener estimaciones agregadas ("gruesas"), de los ingresos a plasmarse en total en la Ley de Ingresos, misma que está conformada por la diversidad de conceptos (impuestos, derechos, productos y aprovechamientos, fundamentalmente).

En este sentido nuestra propuesta suma a la literatura, porque consideramos es factible estimar y describir la recaudación a través de la estadística histórica de la recaudación, de cada ejercicio fiscal, como un todo, y no concepto por concepto, porque la recaudación total, está conformada por los conceptos al interior, y cada uno de ellos ha internalizado los efectos de las variables económicas. Por tanto, al plantear y proponer un modelo, para el cálculo de estimaciones gruesas, tal constituye nuestra aportación.

En cuanto a una técnica y método de trabajo: "metodología", que permita el cálculo de estimaciones agregadas ("gruesas"), en la argumentación se ubica a la interpolación como un método fuerte para determinar valores con un muy alto grado de exactitud (Pacheco-Martínez et al., 2012). Es documentable que se ha apuntado la interpolación de Lagrange o la polinomial de Chebysvev (Zhang et al., 2012), además de la aproximación polinómica por mínimos cuadrados (Gerald y Wheatley, 2000), como métodos de aproximación numérica polinómica que factibilizan el cálculo de recaudación para conceptos puntuales, con bajos márgenes de error (Puls y Gutiérrez, 2014).

Se elige el uso de interpolación polinómica, por ser la opción que facilita la evaluación, diferenciación e integración, en relación con otras opciones, como una serie trigonométrica o exponencial (Pacheco-Martínez et al., 2012).

En este trabajo se propone utilizar la interpolación polinómica de Newton (Diferencias divididas) (De Boor, 2005), para estimar los ingresos fiscales, como opción a las metodologías anteriormente expresadas. 


\section{Metodología}

Se identifica como área de oportunidad que el área de las finanzas públicas que ha alcanzado un menor nivel de desarrollo, substancialmente en cuanto al conjunto de técnicas y métodos de trabajo, "las metodologías" que permiten obtener e

El objetivo buscado se consigue a través del método de caso, esto es, realizando el análisis y discusión de experiencias y situaciones del caso Ciudad de México (CDMX), entidad federativa selecciona por ser la entidad con mayor captación de ingresos propios en México (autosuficiencia financiera) (Salazar et al., 1999).

El desarrollo de la investigación se instruye estableciendo la significación en cuanto a técnicas y métodos de trabajo en la entidad en análisis (CDMX), esto es, las metodologías normadas que habiliten el obtener el producto (la estimación de ingresos), conforme al marco jurídico de la entidad.

Se continua el trabajo con la investigación documental y el estudio escrupuloso de la Cuenta Pública de la CDMX del año 2000 al 2017, para obtener la estadística de los ingresos (estimado, recaudado real) y construir una función tabular con dicha estadística.

Para determinar el polinomio de recaudación, se parte de la representación general de un polinomio de n-ésimo grado.

Se define las ecuaciones de diferencias que sirven para evaluar los coeficientes de la ecuación general del polinomio de interpolación de Newton, en diferencias divididas.

Subsiguientemente se realiza un análisis de los datos, se recupera y estima los valores de la función de recaudación $\mathrm{f}(\mathrm{x})$, que describen la recaudación en cualquier punto del periodo, así como los puntos de cierre (estimación de cierre de un ejercicio fiscal) y el inmediato posterior (estimación del siguiente ejercicio fiscal).

Posteriormente se analiza la información para identificar algún patrón de tendencia que otorgue elementos de validez a los polinomios propuestos como parte de la metodología propuesta. Se determina que efectivamente hay polinomios que arrojan "datos" de estimación razonables, esto al comparárseles con lo reportado en Cuenta Pública (de años para los que los polinomios de diferentes grados proyectan estimación).

Finalmente se concluye que, es factible realizar estimaciones de los ingresos tributarios, a través de la propuesta, tomando como dato insumo la estadística histórica de los ingresos, misma que ha internalizado los efectos de las variables económicas, en entidades con autosuficiencia financiera.

\section{Desarrollo, resultado y discusión}

En México, la Federación, las entidades Federativas y los Municipios, tienen por obligación elaborar y estimar su proyecto de Ley de Ingresos en los términos que refiere el artículo 122 Apartado C, Base Primera, fracción V, inciso b) de la Constitución Política de los Estados Unidos Mexicanos (Cámara de Diputados del H. Congreso de la Unión, 2020).

Ante la masa de posibles casos de estudio en México, es decir, tomar por caso el presupuesto Federal, el de alguna de las 32 entidades Federativas o el de alguno de los 2458 Municipios del país, 
se decide únicamente hacer un análisis, que sea de relevancia en términos de los ingresos propios en el "ente", ya que solo en tales, es relevante hablar de un presupuesto de ingresos (Salazar et al., 1999).

En este sentido, con base a lo reportado en el segundo trimestre de 2018, por ser la información disponible, se encuentra que, en la Ciudad de México, los Ingresos Propios, conformados por la suma de Ingresos Locales más los Ingresos del Sector Paraestatal No Financiero, representaron casi la mitad de los Ingresos del Sector Público Presupuestario de la Entidad (49.6 por ciento), mientras que el restante porcentaje provino de Ingresos de Origen Federal (Gobierno de la Ciudad de México, 2018), siendo por tanto la CDMX la entidad o "ente en México" con mayor autosuficiencia financiera, y por tanto la Entidad Federativa más atractiva, y representativa, para el estudio de su metodología de estimación de metas de recaudación (ingreso), y de ello la elección de la misma para la investigación ( $\sin$ ingresos en el "ente" no es siquiera pensable estimar lo que no se recauda).

Profundizando en la normatividad de la entidad seleccionada, se ubica que en el artículo 40 de la Ley de Presupuesto y Gasto Eficiente de la Ciudad de México (LPGECDMX) (Asamblea Legislativa del Distrito Federal, 2014), se mandata que para estimar el proyecto de Ley de Ingresos, a la base de estimación de cierre a que se refiere la fracción I, del artículo 40 (es decir, a la estimación de los ingresos correspondientes al cierre del ejercicio en curso), se le aplicará, para el caso de actualización de cuotas o tarifas, el factor que para tal efecto se estime de conformidad con el código, refiriéndose por "código" al Código Fiscal de la Ciudad de México vigente (Asamblea Legislativa del Distrito Federal, 2017).

Así, el Código establece como metodología para la estimación del factor, la descrita en el artículo 18, que dicta literal lo siguiente: "en general las cantidades que en su caso se establecen en este Código, que se encuentren vigentes al treinta y uno de diciembre, se actualizarán a partir del primero de enero del año siguiente, conforme a la variación del promedio anual del Índice Nacional de Precios al Consumidor emitido por la autoridad competente al mes de noviembre del año anterior al ejercicio fiscal en que deban actualizarse. Para ello, deberá dividirse el índice promedio de los doce meses más recientes entre el índice promedio de los doce meses anteriores, para aplicar su resultado como factor de ajuste" (Asamblea Legislativa del Distrito Federal, 2017).

Además, el mismo artículo 40 de la LPGECDMX, indica: "Asimismo, con base en las variables económicas se calculará el comportamiento de aquella recaudación en las que por su naturaleza así proceda" (Asamblea Legislativa del Distrito Federal, 2014). Induciendo la idea de que con base en "las variables económicas", exógenas a la recaudación y dependientes de la economía, se habrá de hallar la estimación de recaudación, cuando precisamente "por su naturaleza", puede presumirse que la recaudación en su estadística histórica ya halla internalizado los efectos de las variables económicas al momento, y, por tanto, la estadística de la recaudación es capaz de describirse a sí misma, sin la necesidad de introducir otras variables.

\subsection{Marco Teórico}

De la literatura se ubica que las metodologías usadas por la Federación, las entidades Federativas y los Municipios, y en general cualquier "ente" para la estimación de ingresos varían significativamente. Por ejemplo, es ubicable que la Federación, a través de la Secretaría de Hacienda y Crédito Público, (2019), plantea el uso de modelos estadísticos basados en suavizamientos exponenciales, en donde, 
para la estimación de los modelos estadísticos apunta utilizar series de tiempo mensuales por impuesto, con los cuales se obtienen los factores estacionales por cada impuesto (Secretaría de Hacienda y Crédito Público, 2019). Resumiéndose lo anterior en la realización de estimaciones concepto por concepto de la Ley, lo que no aporta el poder realizar estimaciones gruesas de manera ágil, y sigue lo que la literatura apunta de realizar estimaciones concepto por concepto, pero no de forma agregada (OCDE ET AL., 2020).

Para comprobar y verificar la hipótesis, es decir, que es factible estimar y describir la recaudación, para los proyectos de Ley de ingresos, a través de la estadística histórica agregada de la de recaudación de cada ejercicio fiscal, que ha internalizado los efectos de las variables económicas, en este trabajo se propone utilizar la interpolación polinómica de Newton (Diferencias divididas) (De Boor, 2005), para estimar el proyecto de Ley de Ingresos de la Ciudad de México, como opción a la metodología puntualizada en el artículo 40 de la Ley de Presupuesto y Gasto Eficiente de la Ciudad de México.

Se elige el uso de interpolación polinómica, por ser la opción que facilita la evaluación, diferenciación e integración, en relación con otras opciones, como una serie trigonométrica o exponencial.

Se selecciona utilizar la interpolación polinómica de Newton (diferencias divididas), porque los métodos de diferencia dividida tienen la ventaja de que se pueden agregar más puntos de datos para mejorar la precisión, lo que es especialmente útil cuando se realiza estimaciones en recaudación (en que se puede contar con estadísticas muy detalladas o no, y la interpolación puede ser útil para detallarla o reducirla, o en escenarios, en donde de un momento a otro puede reportarse ideaciones de políticas públicas que se espera contribuyan a mejorar el ingreso de recursos de manera "extraordinaria"), mismos que se pueden incorporar como puntos, sin "desbaratar" las aproximaciones previas, en la búsqueda de la mejor estimación al problema, ganándose precisión en cada iteración de manera ágil. Además, igualmente útil es que, en la interpolación polinómica de Newton, los términos basados en los puntos de datos anteriores se pueden seguir utilizando, lo que abona a la maleabilidad para en un momento dado calibraciones rápidas del modelo.

\subsubsection{Interpolación}

En el campo de las matemáticas conocido como "análisis numérico", los métodos de interpolación son técnicas para construir nuevos puntos de una función a partir de un conjunto discreto de puntos de datos conocidos (Davis, 1975). En este sentido la interpolación es una herramienta matemática ventajosa, que se plantea en este trabajo se use para estimar los valores de recaudación gruesa, en el entendido que para la recaudación fiscal no se tiene una función continua que la describa, sino únicamente los valores de la recaudación que se reportan de manera periódica, como es el caso de la estadística anualizada, reportada en la conocida como Cuenta Pública, en atención al mandato constitucional (Cámara de Diputados del H. Congreso de la Unión, 2016, 2020).

Para manejar la metodología de interpolación de Newton, se propone que los importes de recaudación se interpreten como valores de la función $\mathrm{f}(\mathrm{x})$, específicos e inamovibles para cada 
ejercicio fiscal, el año se interprete como una variable " $\mathrm{x}$ " dada, y para el importe de la recaudación, se considere el comunicado al publicarse la Cuenta Pública de un determinado ejercicio fiscal.

Es así como estos datos de recaudación "se convierten" y representarán funciones tabulares de la siguiente forma:

Tabla 1. Función tabular

\begin{tabular}{|c|r|r|r|r|r|r|}
\hline $\mathrm{x}$ & $\mathrm{x}_{0}$ & $\mathrm{x}_{1}$ & $\mathrm{x}_{2}$ & $\mathrm{x}_{3}$ & $\cdots$ & $\mathrm{x}_{\mathrm{n}}$ \\
\hline $\mathrm{f}(\mathrm{x})$ & $\mathrm{y}_{0}$ & $\mathrm{y}_{1}$ & $\mathrm{y}_{2}$ & $\mathrm{y}_{3}$ & $\cdots$ & $\mathrm{y}_{\mathrm{n}}$ \\
\hline
\end{tabular}

Fuente: Elaboración propia

Donde los valores de "x" representarán los años y los valores de $\mathrm{f}(\mathrm{x})$ lo recaudado en el año correspondiente.

En este sentido, matemáticamente, para estimar una interpolación se requiere la recopilación de los valores de la función $\mathrm{f}(\mathrm{x})$ en los cuales se conocen los argumentos discretos para cada x. Por lo que, como parte de éste trabajo se procedió a la realización de la investigación documental de las Cuentas Públicas, que sucintamente se rescata en la Tabla 2, obteniéndose los datos que son la semilla con los que se busca interpolar, es decir, recuperar y estimar los valores de la función de recaudación $\mathrm{f}(\mathrm{x})$, que describa la recaudación en cualquier punto del periodo, así como los puntos de cierre (estimación de cierre de un ejercicio fiscal) y el inmediato posterior (estimación del siguiente ejercicio fiscal) (Asamblea Legislativa del Distrito Federal, 2014).

Así, para el caso de la Ciudad de México y considerando la Cuenta Pública desde el año 2000 hasta el 2017 (Gobierno de la Ciudad de México, 2001 - 2018), se tiene como datos los valores "x" (años) vs "f(x)", es decir, la recaudación con cifras en millones de pesos, lo que se asienta y puede observarse en la Tabla 2.

Tabla 2. Cuenta pública CDMX 2000-2017 (Cifras en millones de pesos)

\begin{tabular}{|c|c|c|c|}
\hline Año & $\begin{array}{c}\text { Ingresos Totales en millones } \\
\text { de pesos (mdp) }\end{array}$ & Año & $\begin{array}{c}\text { Ingresos Totales en millones } \\
\text { de pesos (mdp) }\end{array}$ \\
\hline $\bar{X}$ & $f(x)$ & $\bar{X}$ & $f(x)$ \\
\hline 2000 & $62,014.19$ & 2009 & $126,621.11$ \\
\hline 2001 & $68,079.62$ & 2010 & $139,305.33$ \\
\hline 2002 & $75,867.85$ & 2011 & $150,974.13$ \\
\hline 2003 & $77,946.94$ & 2012 & $155,768.29$ \\
\hline 2004 & $80,875.02$ & 2013 & $167,775.70$ \\
\hline 2005 & $90,129.30$ & 2014 & $190,386.09$ \\
\hline 2006 & $103,220.26$ & 2015 & $205,062.74$ \\
\hline 2007 & $110,357.93$ & 2016 & $223,089.30$ \\
\hline 2008 & $123,832.30$ & 2017 & $228,081.32$ \\
\hline
\end{tabular}

Fuente: Elaboración propia con datos de Cuentas Públicas 2000-2017 (Gobierno de la Ciudad de México, 2001 - 2018). 
De la anterior Tabla podemos notar que, para un punto, por ejemplo: $\mathrm{x}=2000$, en el año 2002 se tiene que el valor en mdp de $f(x)$ es 62014.19 , es decir, $f(2000)=62014.19$, el cual es un punto conocido de la función de recaudación de la CDMX, perteneciente al conjunto discreto de todos los datos de la recaudación. Estos datos fueron vertidos a la función tabular elaborada, rescatando, uno a uno, el dato reportado en la Cuenta Pública de cada ejercicio fiscal entre 2000 y 2017 y que, en conjunto, describen la recaudación histórica gruesa, esto es, el recaudo en el gran total de los diferentes conceptos que la integran de manera desagregada, como los son: impuestos, derechos, productos y aprovechamientos.

Ahora bien, retomando lo normado en el artículo 40 de la LPGECDMX, es imperativo, que, para cada ejercicio fiscal, se ha de realizar un ejercicio de cierre, que la referida Ley indica como: "estimación de cierre" y la proyección para el siguiente año, que será la Ley de ingresos (Asamblea Legislativa del Distrito Federal, 2014), sin abundar la norma más allá de lo ya referido párrafos atrás. Es de aquí que, siendo propositivos, para realizar lo requerido por la norma, ahora con sustento matemático, se explora novedosamente el usar como método de cálculo la interpolación polinomial, esto porque pese a que es un método de propósito general que existe desde tiempo atrás (De Boor, 2005), su aplicación al cálculo de estimaciones recaudatorias no se ubica en la literatura, por lo que se presume no ha sido explorada.

\subsubsection{Método de Newton por diferencias divididas}

Pese a que existe una gran variedad de métodos matemáticas en los cuales pueden expresarse polinomios de n-ésimo grado, el método de interpolación de Newton en diferencias divididas es uno de los más populares y útiles (De Boor, 2005). Se trata de un método de interpolación polinomial, que consiste en determinar el polinomio único de n-ésimo grado que se ajuste a n+1 puntos. El polinomio definido por este método proporciona una fórmula para calcular cualquier valor de la función, lo que es útil al transferir y aplicar la herramienta matemática, al caso particular de las estimaciones de recaudación, donde se busca calcular los valores de la recaudación de cierre (futuro de corto plazo), así como determinar los valores de proyección hacia el futuro (futuro de mediano plazo).

La propuesta es que, para determinar el polinomio de recaudación, se parta de la representación general de un polinomio de n-ésimo grado, que es:

$$
p_{n}(x)=a_{0}+a_{1}\left(x-x_{0}\right)+a_{2}\left(x-x_{0}\right)\left(x-x_{1}\right)+\ldots+a_{n}\left(x-x_{0}\right)\left(x-x_{1}\right)\left(x-x_{n-1}\right)
$$

Utilizando el polinomio general (ecuación 1), los puntos asociados con los datos de recaudación, Tabla 2, se utilizarán para evaluar los coeficientes $a_{\mathrm{n}}$, tal que, usando $\operatorname{los} x$ datos mencionados, se habrán de resolver las siguientes ecuaciones para determinar cada uno de coeficientes:

$$
\begin{array}{r}
a_{0}=f\left(x_{0}\right) \\
a_{1}=f\left[x_{0}, x_{1}\right]
\end{array}
$$




$$
\begin{gathered}
a_{2}=f\left[x_{0}, x_{1}, x_{2}\right] \\
\vdots \\
a_{n}=f\left[x_{0}, x_{1}, x_{2}, \ldots, x_{n}\right]
\end{gathered}
$$

En donde, la evaluación de la función colocada entre corchetes son lo que se conoce como diferencias divididas finitas y cada una de estas es:

$$
\begin{gathered}
f\left[x_{0}, x_{1}\right]=\frac{f\left(x_{1}\right)-f\left(x_{0}\right)}{x_{1}-x_{0}} \\
f\left[x_{0}, x_{1}, x_{2}\right]=\frac{f\left[x_{1}, x_{2}\right]-f\left[x_{0}, x_{1}\right]}{x_{2}-x_{0}} \\
\vdots \quad \vdots \\
f\left[x_{0}, x_{1}, \ldots, x_{n-1}, x_{n}\right]=\frac{f\left[x_{1}, x_{2}, \ldots, x_{n-1}, x_{n}\right]-f\left[x_{0}, x_{1}, \ldots, x_{n-2}, x_{n-1}\right]}{x_{n}-x_{0}}
\end{gathered}
$$

Estas ecuaciones de diferencias (ecuaciones 6-8) sirven para evaluar los coeficientes de la ecuación general del polinomio de interpolación de Newton, en diferencias divididas (ecuación 1). Así tenemos que, sustituyendo las anteriores, el polinomio general queda expresado de la siguiente manera:

$$
p_{n}(x)=f\left(x_{0}\right)+f\left[x_{0}, x_{1}\right]\left(x-x_{0}\right)+\cdots+f\left[x_{0}, x_{1}, \ldots, x_{n-1}, x_{n}\right]\left(x-x_{0}\right)\left(x-x_{1}\right) \cdots\left(x-x_{n-1}\right)
$$

Cabe hacer mención que, para poder realizar una interpolación de Newton, es necesario que los valores dados en la función tabular $x$ tengan un espaciamiento que sea constante. Lo cual sucede legítimamente en el caso de la recaudación, ya que, tanto en la Cuenta Pública como en la Ley de Ingresos, se reportan datos anualizados (un dato cada 12 meses, que consolida la recaudación de manera inamovible).

Es así como, con lo anterior y definida la Tabla 2 de valores de la función de recaudación, que rescata 18 valores de recaudación, para igual número de años, a través de polinomios de n-ésimo grado (los polinomios únicos de n-ésimo grado que se ajustan a $n+1$ puntos de la función tabular de recaudación), que van desde el más simple de grado uno, hasta el de grado 17, es que se obtendrán por el método de Newton la proyección de cierre y la proyección al siguiente ejercicio.

Con la anterior idea, se logra construir 17 polinomios de newton, que potencialmente deben poder ser útiles para determinar: tanto cualquier ejercicio de cierre, así como la correspondiente proyección para el siguiente año (proyección a futuro).

\subsection{Resultados. Estimación de metas de recaudación mediante polinomios de Newton}

Haciendo uso del método descrito (Newton por diferencias divididas), y dado que se cuenta con los datos de 18 años de recaudación publicados en los documentos que registran las Cuentas Públicas de la Ciudad de México (Gobierno de la Ciudad de México, 2001 - 2018), se pueden determinar 17 polinomios de recaudación, considerando en cada uno de ellos $2,3,4, \ldots$, o hasta 18 puntos 
respectivamente, mismos que se les determinó y con tales se calculó las estimaciones de recaudación asequibles con tales. De lo simulado y calculado se reporta lo registrado en la Tabla 3. Claramente, el número de diferencias divididas es n-1 veces el número de puntos de la función tabular, así para el caso de 18 puntos de la función, las diferencias divididas son 17. Con la intensión de ejemplificar el procedimiento desarrollado para el cálculo de los coeficientes del polinomio y los polinomios mismos, se rescata e indica el caso para 4 puntos y 3 diferencias divididas, que se determinarían sintéticamente de siguiente manera:

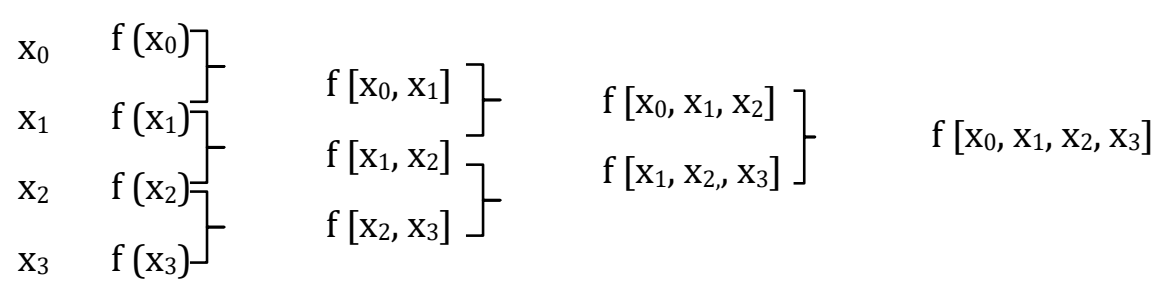

Diferencias divididas que se determinarían con las fórmulas indicadas en la descripción del método, y que fluirán hacia el polinomio de tercer grado siguiente:

$$
p_{3}(x)=f\left(x_{0}\right)+\left(x-x_{0}\right) f\left[x_{0}, x_{1}\right]+\left(x-x_{0}\right)\left(x-x_{1}\right) f\left[x_{0}, ., x_{2}\right]+\ldots
$$

\begin{tabular}{|c|c|c|c|c|c|c|c|c|c|c|c|c|c|c|c|c|c|}
\hline & \multicolumn{17}{|c|}{ ORDEN DEL POLINÓMIO } \\
\hline AÑO & P1 & P2 & P3 & P4 & P5 & $\mathrm{P} 6$ & P7 & $\mathrm{P} 8$ & $\mathrm{Pg}$ & $\mathrm{P} 10$ & P11 & P12 & $\mathrm{P} 13$ & P14 & P15 & P16 & P17 \\
\hline 2018 & $712 E+05$ & $4.348 E+05$ & $-5.630 \mathrm{E}+06$ & $3.718 \mathrm{E}+07$ & $-9.195 \mathrm{E}+07$ & $6.000 \mathrm{E}+07$ & $.985 E+07$ & $9.936 \mathrm{E}+08$ & $-6.418 E+09$ & $1.947 \mathrm{E}+10$ & $3.758 \mathrm{E}+10$ & $4.977 \mathrm{E}+10$ & $-4.571 \mathrm{E}+10$ & $2.852 \mathrm{E}+10$ & $1.148 \mathrm{E}+10$ & $.689 E+09$ & $.792 \mathrm{E}+08$ \\
\hline 2017 & $651 E+05$ & $994 \mathrm{E}+05$ & $-4.654 \mathrm{E}+06$ & $2.864 \mathrm{E}+07$ & $-6.462 E+07$ & $3.668 \mathrm{E}+07$ & $2.437 E+07$ & $5.542 \mathrm{E}+08$ & $-3.152 E+09$ & $355 \mathrm{E}+09$ & $-1.383 E+10$ & $1.528 \mathrm{E}+10$ & $-1.124 E+10$ & $5.258 \mathrm{E}+09$ & $1.410 \mathrm{E}+09$ & $1.651 \mathrm{E}+08$ & $2.281 \mathrm{E}+05$ \\
\hline 2016 & $91 E+05$ & $558 \mathrm{E}+05$ & $-3.796 E+06$ & $2.167 E+07$ & $-4.416 E+07$ & $2.138 \mathrm{E}+07$ & & $2.946 \mathrm{E}+08$ & $-1.449 E+09$ & $289 E+09$ & $-4.542 \mathrm{E}+09$ & & & $6.920 E+08$ & $9.241 E+07$ & $2.231 E+05$ & $2.231 E+05$ \\
\hline 2015 & $30 \mathrm{E}+05$ & $39 \mathrm{E}+05$ & $-3.048 E+06$ & $605 E+07$ & $-2.921 \mathrm{E}+07$ & $1.176 \mathrm{E}+07$ & $83 \mathrm{E}+06$ & $1.479 E+08$ & $-6.150 E+08$ & $162 \mathrm{E}+09$ & $-1.286 \mathrm{E}+09$ & $8.554 E+08$ & $-3.146 \mathrm{E}+08$ & $4.923 E+07$ & $2.051 E+05$ & $2.051 \mathrm{E}+05$ & $2.051 E+05$ \\
\hline 2014 & 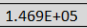 & 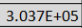 & $-2.402 E+06$ & $160 \mathrm{E}+07$ & $-1.857 E+07$ & & $38 E+06$ & $6.929 \mathrm{E}+07$ & $-2.359 \mathrm{E}+08$ & $E+08$ & $-2.962 E+08$ & $1.319 \mathrm{E}+08$ & \begin{tabular}{l|l|}
$-2.407 \mathrm{E}+07$ \\
\end{tabular} & $1.904 \mathrm{E}+05$ & & & \\
\hline 2013 & $E+$ & $52 \mathrm{E}+05$ & $-1.850 E+06$ & $153 \mathrm{E}+06$ & $-1.124 \mathrm{E}+07$ & $2.802 E+06$ & $716 \mathrm{E}+06$ & $2.977 \mathrm{E}+07$ & $-7.923 \mathrm{E}+07$ & $E+07$ & $-4.986 \mathrm{E}+07$ & $1.131 \mathrm{E}+07$ & $1.678 \mathrm{E}+05$ & $1.678 E+05$ & $1.678 \mathrm{E}+05$ & $1.678 E+05$ & $1.678 E+05$ \\
\hline 2012 & $48 \mathrm{E}+05$ & $85 \mathrm{E}+05$ & $-1.387 \mathrm{E}+06$ & $339 E+06$ & $-6.398 E+06$ & $1.165 \mathrm{E}+06$ & $640 \mathrm{E}+05$ & $1.145 \mathrm{E}+07$ & $-2.209 \mathrm{E}+07$ & $596 \mathrm{E}+07$ & $-4.550 E+06$ & $1.558 \mathrm{E}+05$ & $1.558 \mathrm{E}+05$ & $1.558 \mathrm{E}+05$ & $1.558 \mathrm{E}+05$ & $1.558 \mathrm{E}+05$ & $1.558 \mathrm{E}+05$ \\
\hline 2011 & & & & & & 4.32 & & & & & 1.51 & & & 1.51 & & & \\
\hline 2010 & $\mathrm{E}+05$ & $02 \mathrm{E}+05$ & $-6.916 \mathrm{E}+05$ & $246 E+06$ & $-1.552 E+06$ & $1.673 \mathrm{E}+05$ & $9.130 \mathrm{E}+04$ & $1.072 \mathrm{E}+06$ & $-4.524 \mathrm{E}+05$ & $93 \mathrm{E}+05$ & $1.393 \mathrm{E}+05$ & $1.393 \mathrm{E}+05$ & $1.393 \mathrm{E}+05$ & $1.393 \mathrm{E}+05$ & $1.393 \mathrm{E}+05$ & $1.393 \mathrm{E}+05$ & $1.393 \mathrm{E}+05$ \\
\hline 2009 & 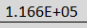 & $86 \mathrm{E}+05$ & $-4.457 \mathrm{E}+05$ & $1.317 \mathrm{E}+06$ & $-5.819 E+05$ & $1.057 E+05$ & $91 \mathrm{E}+04$ & $2.791 \mathrm{E}+05$ & 1.266 & $66 \mathrm{E}+05$ & $1.266 \mathrm{E}+05$ & $1.266 \mathrm{E}+05$ & $.266 \mathrm{E}+05$ & $1.266 \mathrm{E}+05$ & $.266 \mathrm{E}+05$ & $.266 \mathrm{E}+05$ & 1.266E +05 \\
\hline 2008 & $105 E+05$ & $888 \mathrm{E}+05$ & $-2.574 E+05$ & $219 E+05$ & $-1.221 E+05$ & $1.071 E+05$ & $020 E+05$ & $1.238 \mathrm{E}+05$ & & $38 \mathrm{E}+05$ & $1.238 \mathrm{E}+05$ & $1.238 \mathrm{E}+05$ & $.238 \mathrm{E}+05$ & $1.238 \mathrm{E}+05$ & $.238 \mathrm{E}+05$ & $.238 \mathrm{E}+05$ & $1.238 \mathrm{E}+05$ \\
\hline 2007 & $45 E+05$ & $407 \mathrm{E}+05$ & $-1.195 \mathrm{E}+05$ & $702 E+05$ & $5.369 E+04$ & $1.110 E+05$ & $1.104 \mathrm{E}+05$ & $1.104 \mathrm{E}+05$ & thitos & $4 \mathrm{E}+05$ & $1.104 \mathrm{E}+05$ & $1.104 \mathrm{E}+05$ & $1.104 \mathrm{E}+05$ & $1.104 \mathrm{E}+05$ & $.104 E+05$ & $.104 \mathrm{E}+05$ & $1.104 E+05$ \\
\hline 2006 & & & & & & & & & & & $032 E+05$ & $1.032 \mathrm{E}+05$ & $032 E+05$ & & & & $1023=05$ \\
\hline 2005 & $E+04$ & $66 \mathrm{E}+05$ & $3.525 \mathrm{E}+04$ & $52 E+05$ & +04 & $013 E+04$ & $3 E+04$ & $E+04$ & +04 & $E+04$ & $13 E+04$ & $9.013 E+04$ & $013 E+04$ & $9.013 \mathrm{E}+04$ & +04 & $E+04$ & $.013 E+04$ \\
\hline 2004 & & & & & & & & & & & & & & & & & \\
\hline 2003 & & +04 & & & & $E+04$ & +04 & & & & 04 & & $E+04$ & t & & & \\
\hline 2002 & & & & & & & & & & & & & & & & & \\
\hline 2001 & & & & & & $E+04$ & +04 & & & -04 & $308 E+04$ & 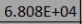 & t & & & & \\
\hline 2000 & $6.201 E+04$ & $6.201 \mathrm{E}+04$ & $6.201 \mathrm{E}+04$ & $6.201 \mathrm{E}+04$ & $6.201 \mathrm{E}+04$ & $6.201 E+04$ & $6.201 \mathrm{E}+04$ & $6.201 \mathrm{E}+04$ & $6.201 \mathrm{E}+04$ & $6.201 \mathrm{E}+04$ & $6.201 \mathrm{E}+04$ & $6.201 \mathrm{E}+04$ & $6.201 \mathrm{E}+04$ & $6.201 E+04$ & $6.201 \mathrm{E}+04$ & $6.201 \mathrm{E}+04$ & $6.201 \mathrm{E}+0$ \\
\hline
\end{tabular}

Tabla 3. Estimaciones de recaudación a través de 17 polinomios de Newton

Fuente: Elaboración propia

Como se aprecia en la tabla 3, se obtuvieron las versiones de los polinomios en diferencias divididas de Newton de primer (P1), segundo (P2), tercer (P3),... y hasta el de grado 17 (P17), mismos que permitieron calcular de manera exacta los puntos de la función tabular (parte inferior de la tabla) y las estimaciones que se reportan en la tabla (parte superior de la tabla).

De lo anterior que se puede afirmar que dichos polinomios efectivamente son capaces de mostrar el comportamiento de la función tabular inicial, acorde al número de puntos considerados, es decir, para el polinomio de grado 1, éste es capaz de dar el resultado exacto en los 2 puntos considerados de la función tabular, para el caso del polinomio de grado 5, éste arroja los 6 puntos de 
la función tabular y lo mismo ocurre para el polinomio de grado 17, toda vez que el mismo es capaz de dar el resultado exacto en los 18 puntos de la función tabular, lo que técnicamente ocurre con todos y cada uno de los polinomios obtenidos(desde el polinomio de primer grado hasta el de grado 17). Lo cual podemos apreciar en la parte inferior de la media pirámide que se forma en la tabla 3 , que asciende desde 2000 a 2018 calculando los puntos de la función tabular interpolados, y que se puede confrontar con lo recolectado y comunicado en la Tabla 2.

Ahora bien, por lo que hace a las estimaciones (parte superior piramidal de la tabla 3), no todos los polinomios arrojan estimaciones que se puedan considerar razonablemente factibles, toda vez que, si bien arrojan un "dato estimado" para un determinado año en lo futuro, al comparárseles con datos de recaudación realmente reportados en Cuenta Pública (toda vez que por fortuna para confrontar, se cuenta con los históricos de recaudación reportados en la Cuenta Pública), lo que se determina del cálculo, indica que no es razonable la estimación. No obstante, de los mismos resultados se puede determinar que efectivamente hay polinomios que arrojan "datos" de estimación razonables, esto al realizar la misma consideración comparativa, esto es, al comparárseles con lo reportado en Cuenta Pública (de años para los que los polinomios de diferentes grados proyectan estimación).

Así los polinomios que arrojan estimaciones probables son el polinomio de primer grado y el de polinomio de segundo grado para la función tabular de la recaudación de la Ciudad de México, que, a saber, con los datos en la función tabular y considerando como vértice el año 2000 son los siguientes:

$$
\begin{gathered}
f_{1}(x)=62,014.19+6,065.43 x \\
f_{2}(x)=62,014.19+6,065.43 x+861.40\left(x^{2}-x\right)
\end{gathered}
$$

\subsection{Discusión}

En el caso práctico del cálculo de: a) un cierre de ingresos y b) una Ley de ingresos del año por venir, la estimación de cierre del ejercicio fiscal se realiza entre agosto y noviembre del año en cuestión (momento del tiempo en que no se dispone del dato exacto del cierre del ejercicio) y la estimación de captación de ingresos del siguiente ejercicio fiscal, igualmente se realiza entre agosto y noviembre del mismo año en que surge (momento del tiempo en que evidentemente sólo es posible hacer una estimación).

Esto es, en base a la información disponible, se requiere necesariamente proyectar un valor para determinar cuáles debieran ser los importes de cierre y de captaciones siguientes o futuras, como lo mandata la propia LPGECDMX (Asamblea Legislativa del Distrito Federal, 2014) para el caso de la CDMX, pero que acontece del mismo modo para cualquier organización.

Así, para probar los polinomios obtenidos, analizando un primer escenario utilizando el polinomio de primer orden y tomando como vértice el año 2014, se calcula y estima los años 2016 y 2017, cuyos resultados se muestran en la tabla 4. Como referencias para probar la calidad de lo arrojado por los polinomios conseguidos, en la columna "Ley de ingresos", se coloca los importes estimados por los administradores en su momento, que se presume fueron calculados con la metodología establecida en el marco normativo, adicional se coloca lo reportado en las "cuentas 
públicas" (reportado en años posteriores, y que son datos con los que hoy día se cuenta, aunque no en aquél momento), que fue el resultado final de la recaudación, el dato duro inamovible de lo realmente recaudado, y finalmente se muestra resaltados en color gris claro, los valores estimados por el polinomio.

Tabla 4. Estimaciones de recaudación 2016-2017 a partir de datos 2014 y 2015

\begin{tabular}{|c|c|c|c|}
\hline AÑO & ESTIMACIÓN & CUENTA PÚBLICA & LEY DE INGRESOS \\
\hline 2017 & $234,416.02$ & $228,081.32$ & $198,965.9771$ \\
\hline 2016 & $219,739.38$ & $223,089.31$ & $181,334.4391$ \\
\hline 2015 & $205,062.74$ & $205,062.74$ & $169,222.6233$ \\
\hline 2014 & $190,386.09$ & $190,386.09$ & $156,837.5765$ \\
\hline
\end{tabular}

Fuente: Elaboración propia con datos de las Cuentas Públicas (Gobierno de la Ciudad de México, 2001 - 2018) y Ley de Ingresos (Gobierno de la Ciudad de México, 2013 - 2018).

Realizada la acotación pertinente en el párrafo que antecede, se encuentra que el polinomio proyecta 219,739.38 mdp para el cierre de 2016, en tanto que para 2017 arroja 234,416.02 mdp. Al compararlas las proyecciones, se tiene que: la proyección de 2016 es 1.52 \% menor respecto a lo realmente recaudado, que son los 223,089.31 mdp registrados en Cuenta Pública de 2016. Para el caso de 2017 lo estimado por el modelo es 2.77 \% mayor que lo realmente recaudado en tal año, que son 228,081.32 mdp. De lo anterior se puede afirmar que ciertamente hay un margen de error de estimación del +/- 3\%, lográndose, a través de estas comparaciones, hacer notar que el modelo arroja estimaciones gruesas que son lo suficientemente razonables.

Ahora bien, comparando las estimaciones obtenidas con el modelo, pero ahora respecto de la Ley de ingresos aprobada por la Asamblea Legislativa de la Ciudad de México, se encuentra lo siguiente: en el caso de la Ley de Ingresos de 2016 la misma está subestimada un $-21.17 \%$, respecto a la estimación arrojada por el modelo, y un -23.02\% respecto de la Cuenta Pública, en el caso del año 2017, igualmente está subestimada la Ley, pero ahora en un $-17.81 \%$ respecto a la estimación que arroja el modelo, y un $-14.63 \%$ respecto a la Cuenta Pública de su ejercicio, lo que refuerza la validez del modelo al mantenerlo en un margen de error de estimación aproximado de +/-3 \%.

Construyendo un segundo escenario, en éste se proyecta el cierre del ejercicio 2018 y la recaudación a captarse en el ejercicio 2019. Así específicamente se calcula y estima los valores para 2018 y 2019, claramente a través del polinomio de primer orden y tomando como vértice el año 2016. Los valores obtenidos a través del polinomio se reportan y se muestran en la Tabla 5, así como los propios de la Cuenta Pública y los de la Ley de Ingresos que a la fecha están disponibles, toda vez que se reportan por Ley meses después de concluidos los ejercicios fiscales. 
Tabla 5. Estimaciones de recaudación 2018-2019 a partir de datos 2016 y 2017

\begin{tabular}{|r|r|r|r|}
\hline AÑO & ESTIMACIÓN & $\begin{array}{c}\text { CUENTA } \\
\text { PÚBLICA }\end{array}$ & $\begin{array}{c}\text { LEY DE } \\
\text { INGRESOS }\end{array}$ \\
\hline 2019 & $238,065.33$ & $\begin{array}{c}\text { No } \\
\text { disponible }\end{array}$ & $234,000.16$ \\
\hline 2018 & $233,073.33$ & $\begin{array}{c}\text { No } \\
\text { disponible }\end{array}$ & $226,851.90$ \\
\hline 2017 & $234,416.02$ & $228,081.32$ & $198,965.98$ \\
\hline 2016 & $219,739.38$ & $223,089.31$ & $181,334.44$ \\
\hline
\end{tabular}

Fuente: Elaboración propia con datos de las Cuentas Públicas (Gobierno de la Ciudad de México, 2001 - 2018) y Ley de Ingresos (Gobierno de la Ciudad de México, 2013 - 2018).

Se encuentra que el polinomio proyecta 233,073.33 mdp para el cierre de 2018, en tanto que para 2019 arroja 238,065.33 mdp. Estas proyecciones pueden darse por legítimas dada la validez mostrada por el modelo de diferencias divididas de Newton usado en el primer escenario propuesto, y que es el mismo que se utilizó para el cálculo de estas últimas dos estimaciones. Corroborar estas estimaciones, sólo será posible en el momento en que sean publicados los datos de lo realmente recaudado y registrado en la Cuenta Pública de tales años, lo que, al momento de la elaboración de este manuscrito, no se ha dado.

Con la información disponible para comparación, se puede decir que, para el caso del cierre del 2018, lo estimado por el modelo es $2.74 \%$ mayor que la meta a recaudar impuesta para el año, por el ahora Congreso de la Ciudad de México, que son 226,851.90 mdp, por lo que ciertamente el modelo continúa manteniéndose en un margen de error de estimación de $+/-3 \%$, reafirmándose que el modelo arroja estimaciones gruesas lo suficientemente razonables.

\section{Recomendaciones, limitaciones e implicaciones}

Se recomienda el uso de la metodología para la proyección y el análisis de los ingresos tributarios en "entes" con captación-autosuficiencia financiera.

Dada la disponibilidad de datos, sin limitación para la realización de la investigación en el caso seleccionado, se ubica que la principal limitación de la aplicación de la metodología a las entidades federativas y municipios radica en que la recaudación está centralizada y no tienen autosuficiencia financiera.

\section{Originalidad}

Esta investigación es original dado que aporta una metodología de trabajo al sub-área disciplinar del área de las finanzas públicas, misma que se le ubica con un bajo nivel de desarrollo en cuanto a técnicas y métodos para la proyección y el análisis de los ingresos tributarios de manera agregada, metodología de trabajo implementable por las sub-áreas de ingresos tributarios en la referida área de finanzas públicas. 


\section{Conclusiones y consideraciones finales}

Finalmente se concluye que, es factible realizar estimaciones de los ingresos tributarios, a través de la estadística histórica de los ingresos que han internalizado los efectos de las variables económicas, lo que puede ser adoptado como política pública.

Además se concluye que si bien, cualquier entidad federativa puede beneficiarse con el uso de la metodología propuesta, para la estimación de la recaudación pública, es importante hacer la consideración que es particularmente útil en entidades con autosuficiencia financiera, no siendo por tanto aplicable a entidades federativas y/o municipios en los cuales hay una baja disposición de ingresos propios, puesto que no cuentan con datos, ni presentes ni históricos de recaudación propia, que internalice los efectos de las variables económicas y que logre describir sus ingresos.

Igualmente se concluye que la metodología planteada es útil para aquellas áreas de las finanzas públicas que deseen mejorar o reformular los procedimientos de trabajo actuales, utilizar nuevos enfoques en viejas tareas o, mejor aún, modificar los procedimientos legislados, a partir de una metodología basada en la estadística histórica que internaliza la visión del conjunto de las cuestiones inherentes a ella.

Particularmente en este trabajo se define, simula, demuestra y concluye, que es totalmente factible la estimación de recaudación hacendaria mediante la interpolación polinómica por el método de las diferencias divididas de Newton, considerando la recaudación como una función tabular que se ajustará polinómicamente, tal que se emplee el polinomio perfilado para fijar las metas de recaudación en proyectos de presupuesto en lo general, y en particular, en los proyectos de Ley de Ingresos.

Al mismo tiempo, esta investigación sienta las bases para poder usar el método matemático de las diferencias divididas de Newton, en proyectos de presupuesto de entes con autosuficiencia financiera, como opción a las metodologías puntualizadas en las diversas Leyes de Presupuesto: de la Federación, de las entidades Federativas y los Municipios, en México, basadas en factores de actualización u otras de naturaleza similar.

Además, esta investigación logra comprobar empíricamente, la hipótesis, es decir, que es realizable estimar y describir la recaudación a través de la estadística histórica de la de recaudación de cada ejercicio fiscal, que ha internalizado los efectos de las variables económicas.

De lo analizado y explorado en esta investigación para el caso estudiado, Ciudad de México, es posible afirmar que, usando un polinomio de Newton de primer o segundo grado, se obtiene interpolaciones (polinomios) que permiten hacer estimaciones, con un margen de error de $+/-3 \%$, lográndose proyecciones gruesas que son lo suficientemente razonables.

Como trabajo a futuro, mismo que potencialmente podría ajustar mejor las estimaciones obtenidas a través del arreglo propuesto, se visualiza, plantear otros escenarios, ahora tal vez considerando disminuir el importe de los datos colectados en la estadística histórica de la recaudación, en importes que representen el cómo se hace sentir en la recaudación los "efectos" de las variables endógenas, es decir, las variables que representan disminución o aumento de la recaudación, que bien se pudieran establecer coloquialmente como los efectos monetarios -en dinero- de los programas o "estrategias de recaudación" implementados por la administración en un 
determinado ejercicio fiscal, que se forman o engendran en el interior de las Tesorerías, es decir, se originan por causas de las políticas de recaudación que se instrumentan, como lo son los programas de regularización fiscal, los programas y resoluciones de carácter general para el otorgamiento de subsidios o condonaciones a los contribuyentes, y que claramente repercuten en los importes totales, de los importes gruesos de recaudación obtenidos en un ejercicio u otro, por la existencia o no de los mismos, acorde con la política fiscal.

\section{Referencias}

[1] Andino Alarcón, M., Parra, J. C. (2007). Estimación de la brecha de recaudación del IVA e Impuesto a la Renta de las sociedades por industria. Revista Institucional del Servicio de Rentas Internas. (1), 101 139.

[2] OCDE et al. (2020), Estadísticas tributarias en América Latina y el Caribe 2020, OECD Publishing. doi:|10.1787/68739b9b-en-es

[3] Contreras, C., \& Contreras, M. (2015). Estimación de la recaudación del impuesto sobre transacciones financieras: el caso español. Hacienda Pública Española/Review of Public Economics, 213(2), 109-143. doi:|10.7866/hpe-rpe.15.2.5

[4] Davis, Philip J. (1975), Interpolation and approximation. Courier Corporation. doi:|10.1137/1007028

[5] De Boor, C. (2005). Divided differences, Surveys in Approximation Theory, (1), 46-69. doi:|10.1017/cbo9781139171502.006

[6] Gracia, J. J. E., \& Chueca, J. A. M. (2000). Recaudación fiscal derivada del consumo de tabaco en España: estimaciones a partir de un modelo de adicción racional. Hacienda pública española, (153), 45-52.

[7] Gerald, C., \& Wheatley, P. (2000). Análisis numérico con aplicaciones. Pearson Educación.

[8] Jarach, D. (2013). Finanzas públicas y derecho tributario. AbeledoPerrot.

[9] Martín, F. R. (2005). El Método directo de proyección de los ingresos tributarios. Un modelo para su aplicación. Revista de la Asociación Internacional de Presupuesto Público, (57).

[10] Martín, F. R. (2009). La economía de los ingresos tributarios. Un manual de estimaciones tributarias. CEPAL.

[11] Pacheco-Martínez, N. J., Juárez-Toledo, C., \& Martinez-Carrillo, I. (2012). Análisis dinámico de la estabilidad usando interpolación de alto orden. Ingeniería, investigación y tecnología, 13(4), 451-460. doi:|10.22201/fi.25940732e.2012.13n4.044

[12] Rossignolo, D. (2012). Estimación de la recaudación potencial del impuesto a la renta en América Latina. CEPAL.

[13] Salazar, J. I., Musi, A. S., \& Cervantes, L. S. (1999). México: Ingresos estatales y dependencia de las participaciones federales. Comercio exterior.

[14] Puls, S. L. (2014). La recaudación del impuesto al hospedaje en el estado de Quintana Roo, México: estadística descriptiva y patrón de tendencia fractal. Cuadernos de Economía, 37(103), 45-51. doi:|10.1016/j.cesjef.2013.11.001

[15] Puls, S. L., \& Gutiérrez, G. A. (2014). Métodos polinómicos como estimadores para la recaudación fiscal: caso aplicado al impuesto sobre nóminas en Quintana Roo, México. Cadernos PROLAM/USP, 13(24), 61-76. doi:|10.11606/issn.1676-6288.prolam.2014.88781

[16] Salim, J., D’Angela, W., Puente, M. M. P., \& Mancini, D. M. (2005). Estimación del incumplimiento en el IVA. Años 2000 a 2004. Informe de Recaudación Segundo trimestre de 2005.

[17] Zhang, X., Guo, X., \& Xu, A. (2012). Computations of fractional differentiation by Lagrange interpolation polynomial and Chebyshev polynomial. Information Technology Journal, 11(4), 557. doi:|10.3923/itj.2012.557.559 


\section{Fuentes documentales}

[18] Asamblea Legislativa del Distrito Federal, (2017). Código Fiscal del Distrito Federal, Instituto de Investigaciones Parlamentarias, México.

[19] Asamblea Legislativa del Distrito Federal, (2014). Ley de Presupuesto y Gasto Eficiente del Distrito Federal, Instituto de Investigaciones Parlamentarias, México.

[20] Cámara de Diputados del H. Congreso de la Unión (2020). Constitución Política de los Estados Unidos Mexicanos. Diario Oficial de la Federación.

[21] Cámara de Diputados del H. Congreso de la Unión (2016). Ley de Fiscalización y Rendición de Cuentas de la Federación, Secretaría de Servicios Parlamentarios.

[22] Gobierno de la Ciudad de México, (2001). Cuenta Pública del D.F. 2000, Ciudad de México.

[23] Gobierno de la Ciudad de México, (2002). Cuenta Pública del D.F. 2001, Ciudad de México.

[24] Gobierno de la Ciudad de México, (2003). Cuenta Pública del D.F. 2002, Ciudad de México.

[25] Gobierno de la Ciudad de México, (2004). Cuenta Pública del D.F. 2003, Ciudad de México.

[26] Gobierno de la Ciudad de México, (2005). Cuenta Pública del D.F. 2004, Ciudad de México.

[27] Gobierno de la Ciudad de México, (2006). Cuenta Pública del D.F. 2005, Ciudad de México.

[28] Gobierno de la Ciudad de México, (2007). Cuenta Pública del D.F. 2006, Ciudad de México.

[29] Gobierno de la Ciudad de México, (2008). Cuenta Pública del D.F. 2007, Ciudad de México.

[30] Gobierno de la Ciudad de México, (2009). Cuenta Pública del D.F. 2008, Ciudad de México.

[31] Gobierno de la Ciudad de México, (2010). Cuenta Pública del D.F. 2009, Ciudad de México.

[32] Gobierno de la Ciudad de México, (2011). Cuenta Pública del D.F. 2010, Ciudad de México.

[33] Gobierno de la Ciudad de México, (2012). Cuenta Pública del D.F. 2011, Ciudad de México.

[34] Gobierno de la Ciudad de México, (2013). Cuenta Pública del D.F. 2012, Ciudad de México.

[35] Gobierno de la Ciudad de México, (2014). Cuenta Pública del D.F. 2013, Ciudad de México.

[36] Gobierno de la Ciudad de México, (2015). Cuenta Pública del D.F. 2014, Ciudad de México.

[37] Gobierno de la Ciudad de México, (2016). Cuenta Pública del D.F. 2015, Ciudad de México.

[38] Gobierno de la Ciudad de México, (2017). Cuenta Pública del D.F. 2016, Ciudad de México.

[39] Gobierno de la Ciudad de México, (2018). Cuenta Pública del D.F. 2017, Ciudad de México.

[40] Gobierno de la Ciudad de México, (2018). Informe de Avance Trimestral Enero-Junio 2018, Ciudad de México.

[41] Gobierno de la Ciudad de México, (2012). Ley de ingresos CDMX 2013, Ciudad de México.

[42] Gobierno de la Ciudad de México, (2013). Ley de ingresos CDMX 2014, Ciudad de México.

[43] Gobierno de la Ciudad de México, (2014). Ley de ingresos CDMX 2015, Ciudad de México.

[44] Gobierno de la Ciudad de México, (2015). Ley de ingresos CDMX 2016, Ciudad de México.

[45] Gobierno de la Ciudad de México, (2016). Ley de ingresos CDMX 2017, Ciudad de México.

[46] Gobierno de la Ciudad de México, (2017). Ley de ingresos CDMX 2018, Ciudad de México.

[47] Secretaría de Hacienda y Crédito Público, (2019). Metodología de la estimación de los ingresos anuales y mensuales. Proyecto de Presupuesto de Egresos de la Federación. 